\title{
Analysis of PMP Module Using Mesh Generation Techniques
}

\author{
Joon Seong Lee* \\ Dept. of Mechanical System Engineering \\ Kyonggi University \\ 154-42, Gwanggyosan-ro, Yeongtong-gu, Suwon, Gyeonggi-do, 443-760 Korea \\ *Corresponding author
}

\begin{abstract}
Consumers are generally required the use of high energy density batteries. However, most consumers are often dissatisfied with the battery life from even the most advanced lithium-ion rechargeable batteries in mobile phone. All In recent years, plastic products have become thinner and lighter and proper materials, processing technology and product technology have been developed accordingly. PMP (Protection Module Package) has the potential of providing energy densities that are several times more than that PCM (Protection Circuit Module), making them attractive power source for mobile applications, such as next generation cellular phones that require high energy density power sources to enable extended operation times. In this paper, we explain an element generation method of PMP module using several types. Also, it is performed the several analysis of epoxy molding compound module using this mesh generation method.
\end{abstract}

Keywords-component; protection circuit module, protection module package, mesh generation, finite element method

\section{INTRODUCTION}

The mesh generation process, which influences computational accuracy as efficiency and whose fully automation is very difficult in three-dimensional cases, has become the most critical issue in a whole process of the FE analyses. In this respect, one of the authors has been developed CAE system with mesh generation [1].

Consumers are generally required the use of high energy density batteries. However, most consumers are often dissatisfied with the battery life from even the most advanced lithium-ion rechargeable batteries in mobile phone[2]. In recent years, plastic products have become thinner and lighter and proper materials, processing technology and product technology have been developed accordingly. PMP (Protection Module Package) has the potential of providing energy densities that are several times more than that PCM (Protection Circuit Module),3 making them attractive power source for mobile applications, such as next generation cellular phones that require high energy density power sources to enable extended operation times

In this paper, several mesh generation method which is useful automated analysis system are describe. Also, FE analysis simulation for PMP module was carried out using several mesh generation method.

\section{System Using BubBle PACKING}

The developed CAE system allows designers to evaluate detailed physical behaviors of structures through some simple interactive operations to their geometry models. In other words, designers do not have to deal with mesh data when they operate the system. The key element of this system, bubble meshing[4] lies in the first step, that is, the optimization of mesh node locations by close packing bubbles. By this method, bubbles move in a domain until forces between them are stabilized, and Delaunay triangulation is then applied to generate a mesh connecting the nodes defined by the bubble packing. A repulsive or attractive force much like an intermolecular van der Waals force is assumed to exist between two adjacent bubbles.

A globally stable configuration of tightly packed bubbles is determined by solving the equation of motion. The novelty of this method is that the close packing of bubbles forms a pattern of Voronoi polygons, corresponding to well-shaped Delaunay triangles[5]. Figure 1 shows the procedure of the bubble packing method. Bubble meshing generates a twodimensional triangular mesh by the following two steps: (a) Solving the equation of motion on vertices, edges, and faces (or loops) in that order, (b) Generation of triangular mesh by connecting the center points of bubbles by Delaunay Triangulation. Similar steps are also applied to the generation of three-dimensional tetrahedral meshes.
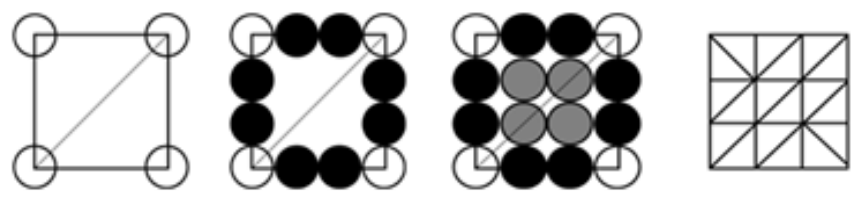

FIGURE I. PROCEDURE OF THE BUBBLE MESH

\section{NODE GENERATION}

Node generation is one of time consuming processes in automatic mesh generation. The input data required are only the node density of the base node pattern such as unit distance of nodes, the kinds of special node patterns, and the location and node densities at the representative points of the special node patterns. 
The procedure of two dimensional node generation of the base node pattern is illustrated in Figure 2. First, either a circumscribed rectangle or box (in the 3D) to the domain is determined, in which nodes are generated regularly. A distance of neighboring nodes of the pattern, which is called "base grid size" here, is inputted by a user.

Second, each node is examined whether to be inside the domain by the IN-OUT check criterion, and any nodes outside the domain are removed.

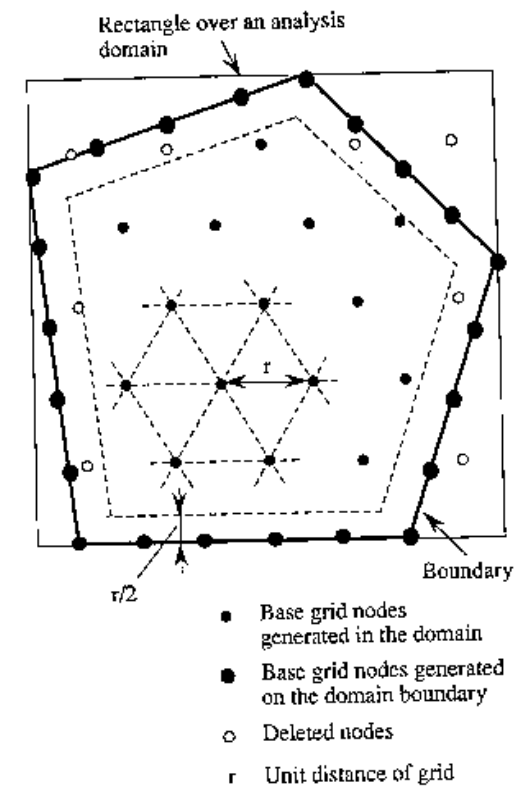

FIGURE II. GENERATION OF BASED NODE PATTERN

Any nodes located very closely to the domain boundary are removed as well to avoid undesirable distortion of mesh shape near the domain boundary. Among algorithms for uniform node generation, the present method may not be the best one as for the uniformity of node locations near the domain boundary, but it is very simple operation. Next, several node patterns are generated through an interactive operation between a user and the system. The input data required here are only the node density and the location of each stress concentration point.

\section{Meshless Method}

Recent advance in computer technology has enabled a number of complicated natural phenomena to be simulated, which were only observed by experiments. Meshless method [6] has the algorithms described in Figure 3. The method commences with the appropriate allocation of nodes in the domain to be analyzed $\Omega$. We first give nodal points $\operatorname{Pi}(\mathrm{x}, \mathrm{y})$, $\forall \mathrm{i} \in\{1, \ldots, \mathrm{n}\}$, in the domain with radius ri, which represents nodal density, as illustrated in Figure 4.

Meshless method then allows each node Pi to nominate a set of candidate nodes for creating local elements within radius $r_{i}$, altogether being $C_{i}$, which is given by

$$
\begin{aligned}
\mathrm{C}_{\mathrm{i}} & =\left\{P_{j} \mid \overline{P_{1} P_{j}}<r_{i,} \forall j \in\left\{1_{x, n} n\right\}\right\}, \\
& \equiv\left\{P_{0}^{c}, P_{1, \ldots,}^{c}, P_{n}^{e}\right\}
\end{aligned}
$$

where $P_{0}^{c}=P_{1}$ is the current central node, $n_{c}$ is the number of candidate nodes $\left(n_{c}<n\right)$ and

$$
\overline{P_{1} P_{j}}=\sqrt{\left(x_{\mathrm{i}}-x_{j}\right)^{2}-\left(y_{\mathrm{i}}-y_{j}\right)^{2}} \text {. }
$$

Subscript c indicates that the numbering of each node is on a local basis.

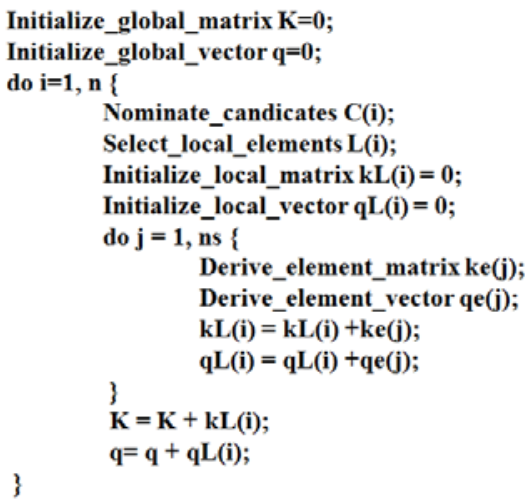

FIGURE III. BASIC ALGORITHM OF MESHLESS METHOD

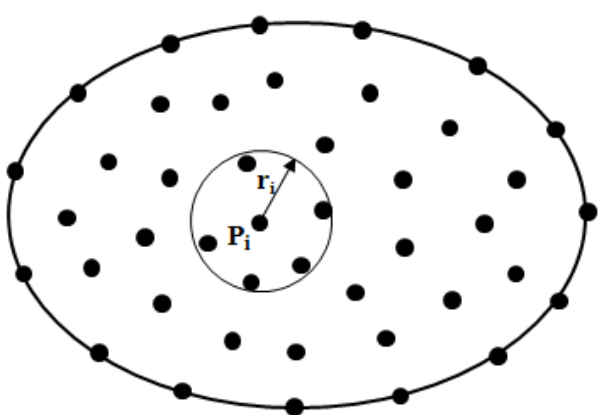

FIGURE IV. FCENTRAL NODE AND SATELLITE NODE

Local elements are each then selected by connecting two points $P_{\mathscr{Q}}^{C}$ and $P_{\mathscr{b}}^{\mathscr{C}}$ with the center $P_{0}^{C}$.

The important note in the selection is that local elements created at each node must be consistent in a global manner. Figure 5 illustrates and example of violation in the selection, where element $\triangle P_{a} P_{b} P_{d}$, created with central node $P_{a}$ does not coincide with the equivalent $\triangle P_{d} P_{a} P_{c}$ having $P_{d}$ as its center. This inconsistency can be eliminated by setting up rules that select local elements irrespective of what the current central node is. To begin with, all $\Delta P_{i}^{E} P_{j}^{E} P_{k}^{C}$ are considered to be local elements. $P_{j}^{C} P_{k}^{c}$ is then sorted in its length and, from the shortest, eliminative rules described in Figure 6 are carried out. Here we will describe the set of nodes in Figure 7, which finally construct the local elements, for further convenience as follows: 


$$
\mathrm{L}_{\mathrm{i}}=\left\{P_{0}^{g}, P_{1, \ldots,}^{g}, P_{\mathrm{n}}^{g}\right\}
$$

where the subscript $s$ indicates that each node is numbered with the selected local elements.

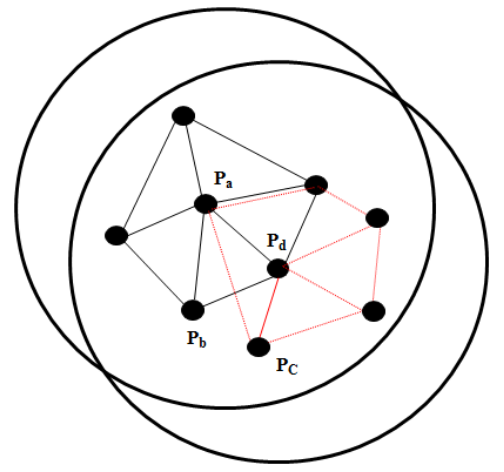

FIGURE V. EXAMPLE OF VIOLATION IN LOCAL ELEMENTS SELECTION

\section{if there is a point in $\triangle \mathrm{PiP}_{\mathbf{i}} \mathrm{Pk}_{\mathrm{k}}$ eleminate $\triangle \mathbf{P i P j}_{\mathbf{j}} \mathbf{P}$;}

else \{

if there is $\mathbf{P}_{\mathrm{i}} \mathbf{P}_{\mathbf{x}}$ intersecting with $\mathrm{P}_{\mathrm{j}} \mathbf{P}_{\mathrm{k}}$ if $\left(\min \left\{\mathbf{P i}_{\mathbf{i}} \mathbf{P}_{\mathbf{x}}\right\}>\mathbf{P}_{\mathbf{j}} \mathbf{P}_{k}\right)$ else eliminate al $P_{x}$; eliminate $\triangle \mathbf{P i}_{\mathbf{i}} \mathbf{P}_{\mathbf{k}}$;

\}

FIGURE VI. SELECTION OF LOCAL ELEMENTS

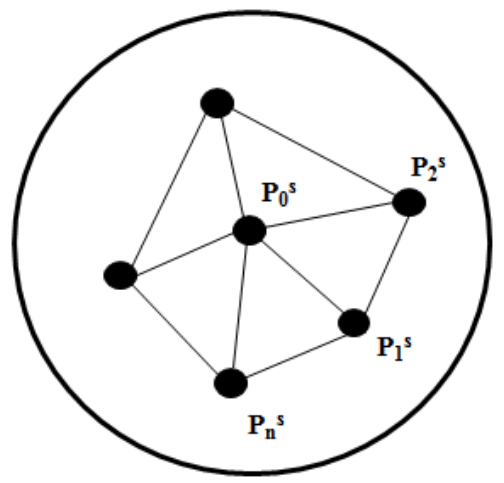

FIGURE VII. A SET OF LOCAL ELEMENTS

\section{PMP MODULE}

PMP module is introduced to reduce the thickness and internal resistance of the mobile phone battery. Figure 8 shows the shape of PMP module. Figures 9 and 10 is a configuration model and modeling for heat transfer analysis of PMP module respectively. In this study, a protection module of mobile battery was chosen because it is the flat plate of small thin wall which is expected to have great short shot and flexing. The size of module $3 \mathrm{~mm} \times 12 \mathrm{~mm} \times 1 \mathrm{~mm}$ was used in this evaluation. Table 1 shows the property of different epoxy molding.

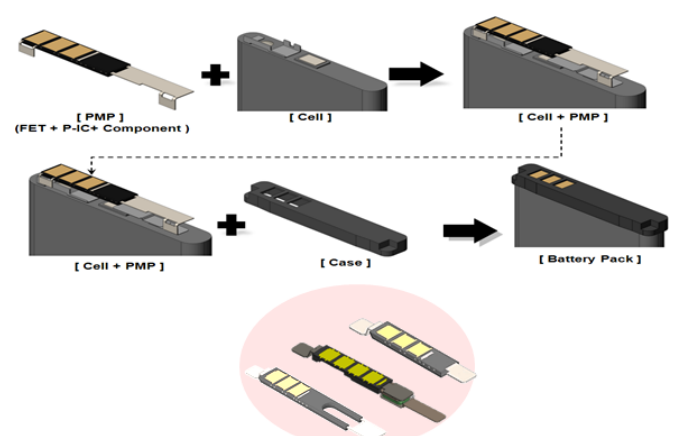

FIGURE VIII. SHAPE OF PMP MODULE

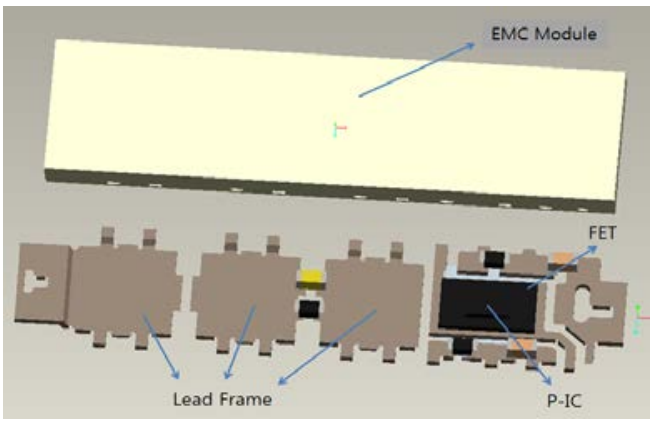

FIGURE IX. CONFIGURATION OF MODEL

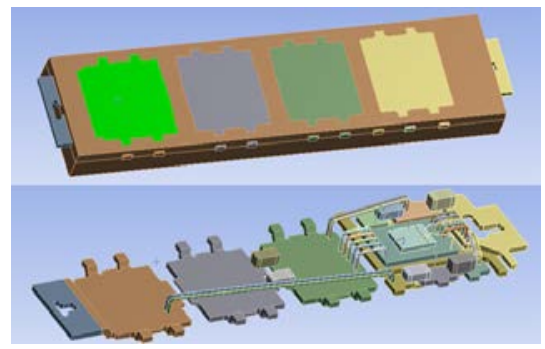

FIGURE X. MODELING FOR ANALYSIS

TABLE I. PROPERTY OF SEVERAL MATERIAL

\begin{tabular}{|c|c|c|c|}
\hline Type & A & B & C \\
\hline Density[g/cc] & 1.82 & 1.88 & 1.79 \\
\hline $\begin{array}{c}\text { Flexural } \\
\text { Modulus[GPa] }\end{array}$ & 12 & 16 & 17 \\
\hline Poisson' ratio & 0.43 & 0.36 & 0.34 \\
\hline
\end{tabular}

\section{EXAMPLES AND DISCUSSIONS}

The performance of the mesh generator is demonstrated through the mesh generation of several geometries. In case of a complex geometry as shown in Figure 11, a uniform mesh and a nonuniform mesh were connected very smoothly. Bubble and elements are generated in about 2 minutes and in about 3 minutes, respectively. The mesh consists of 6.896 tetrahedral elements.

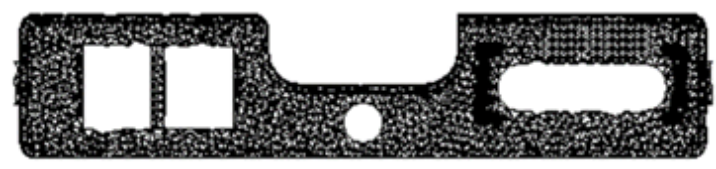

FIGURE XI. BUBBLE MESH 


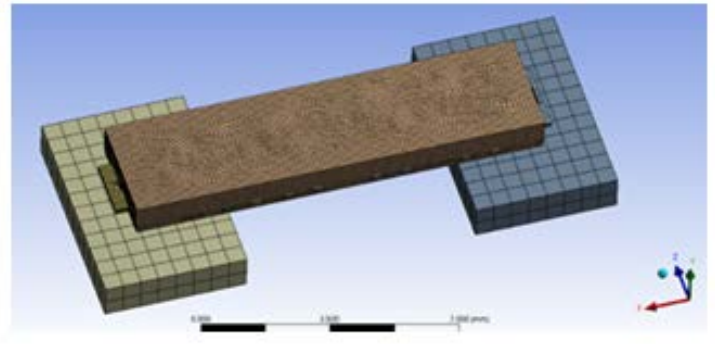

FIGURE XII. DELAUNAY MESH OF PMP
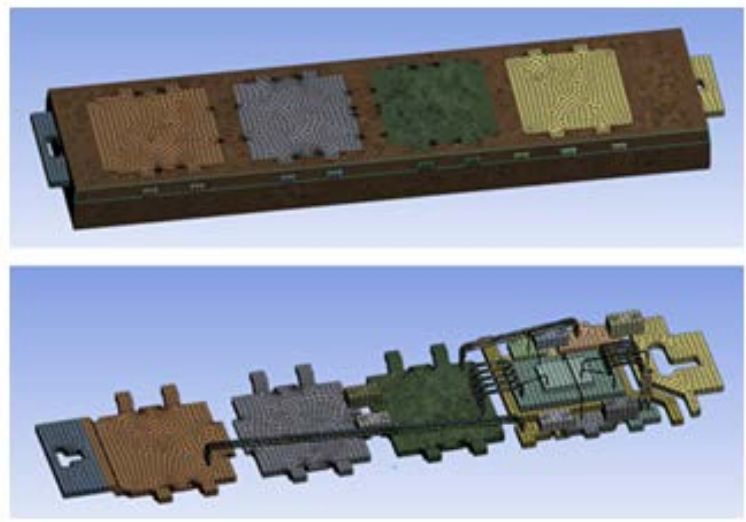

FIGURE XIII. MESH FOR HEAT TRANSFER ANALYSIS

Figure 12 and Figure 13 is a mesh for strength analysis and thermal analysis of PMP module respectively.

The several mesh generation method was applied to static heat conduction problem of three type's battery to show the performance and accuracy. Table 2 shows the comparisons of temperature values obtained at four points a, b, c and d along the top edge of the domain between the several mesh generation method.

The nodal patterns are equally applied. This means that a mesh for this domain is created implicitly by this method and that the small difference between the present solution and that of the Delaunay mesh attributes to the difference of the mesh topology seen at a few parts of the domain between the three methods.

TABLE II. ANALYSIS RESULT FOR TEMPERATURE

\begin{tabular}{|c|c|c|c|c|}
\hline Material & Location & $\begin{array}{c}\text { Bubble } \\
\text { Mesh }\end{array}$ & $\begin{array}{c}\text { Delaunay } \\
\text { Mesh }\end{array}$ & $\begin{array}{c}\text { Meshless } \\
\text { Method }\end{array}$ \\
\hline \multirow{4}{*}{ A } & $\mathrm{a}$ & 20.451 & 20.452 & 20.456 \\
\cline { 2 - 5 } & $\mathrm{b}$ & 22.463 & 22.463 & 22.464 \\
\cline { 2 - 5 } & $\mathrm{c}$ & 30.525 & 30.527 & 30.529 \\
\cline { 2 - 5 } & $\mathrm{d}$ & 49.884 & 49.884 & 49.887 \\
\hline \multirow{4}{*}{ B } & $\mathrm{a}$ & 19.251 & 19.251 & 19.254 \\
\cline { 2 - 5 } & $\mathrm{b}$ & 21.243 & 21.244 & 21.246 \\
\cline { 2 - 5 } & $\mathrm{c}$ & 29.219 & 29.220 & 29.223 \\
\cline { 2 - 5 } & $\mathrm{d}$ & 48.638 & 48.639 & 48.640 \\
\hline \multirow{4}{*}{ C } & $\mathrm{a}$ & 17.360 & 17.360 & 17.362 \\
\cline { 2 - 5 } & $\mathrm{b}$ & 20.822 & 20.823 & 20.826 \\
\cline { 2 - 5 } & $\mathrm{c}$ & 28.407 & 28.408 & 28.409 \\
\cline { 2 - 5 } & $\mathrm{d}$ & 47.647 & 47,648 & 47.650 \\
\hline
\end{tabular}

\section{CONCLUSION}

The In this paper, practical performance of battery using several mesh generation methods such as bubble meshing, Delaunay triangulation method and meshless method is demonstrated through heat conduction analysis. It is shown that nearly the same solution as the conventional finite element method using the Delaunay mesh is obtained under the condition of distribution pattern of nodes. Also, a technique, incorporating patch elements to achieve in meshless method, has been proposed. The use of patch elements can reduce the size of the global stiffness matrix in comparison to that by quadrilateral elements, as the quantity of the central node at each patch element is dependent on those at its vertices. It is shown that the meshless technique was used to solve a steady state thermal conductivity problem and the same problem was solved with the normal finite element method for comparison.

\section{REFERENCES}

[1] J.S. Lee, “An Automated CAE System for Three-Dimensional Complex Geometry,” Doctoral Thesis, The University of Tokyo, 1995. J. Clerk Maxwell, A Treatise on Electricity and Magnetism, 3rd ed., vol. 2. Oxford: Clarendon, 1892, pp.68-73.

[2] J.S. Lee, H.R. Hong, G.H. Jo and D.K. Park, "Strength Analysis of Epoxy Molding Compound Module Using Automated FEA System," Applied Mechanics and Materials, Vol. 670-671, pp. 852-855, 2014

[3] J.S. Lee, "Automated Structural Design System Using Fuzzy Theory and Neural Network," Int. J. of the Korean Society of Precision Engineering, Vol. 3, No. 1, pp. 43-48, 2002

[4] J.S. Lee, "Mesh Generation Methodology for FE Analysis of 3D Structures Using Fuzzy Knowledge and Bubble Method,” J. of Korean Institute of Intelligent Systems, Vol. 19, No. 2, pp. 230-235, 2009.

[5] J.S. Lee, "Development of High-Performance FEM Modeling System Based on Fuzzy Knowledge Processing,” Int. J. of Fuzzy Logic and Intelligent Systems, Vol. 4, No. 2, pp. 193-198, 2004.

[6] J.S. Lee, "Meshless Finite Element Analysis of Three-Dimensional Problems Using Fuzzy Knowledge Processing,” J. of Fuzzy Logic and Intelligent Systems, Vol. 4, No. 4, pp. 1-7, 1998. 